\title{
Eventos adversos em Cuidados de Saúde Primários: promover uma cultura de segurança
}

Maria José Ribas*

\section{RESUMO}

A segurança do utilizador dos serviços de saúde não tem sido, até à data, sistematicamente incluída nas prioridades de quem organiza e presta cuidados, em particular no âmbito dos Cuidados de Saúde Primários.

Este conceito, que não é novo, inclui um vasto conjunto de acções que visam evitar a ocorrência de eventos adversos para o doente: danos, quase-falhas, incidentes, acidentes ou erros.

Os factores que contribuem para a ocorrência de um evento adverso podem ser múltiplos e podem ser prevenidos.

Para tal é necessário conhecê-los, antecipá-los, preveni-los e, quando ocorrem, notificá-los, discuti-los, partilhá-los e aprender com eles.

É urgente implementar mudanças que promovam uma cultura de segurança e tornem todas as actividades de cuidado aos doentes em Cuidados de Saúde Primários, desde a entrada até à saída do sistema, seguras e redutoras do risco de sofrer eventos adversos.

Palavras-chave: Eventos Adversos; Segurança do Doente; Erro Médico; Cuidados de Saúde Primários.

«Talvez pareça estranho enunciar como primeiro dever de um hospital não causar mal ao paciente.»

Florence Nightingale, 1859

«First, do not harm»

Atribuído a Hippocrates, circa 470-360 ac

\section{INTRODUÇÃO}

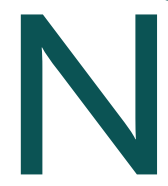

as últimas décadas, a segurança do doente tem sido mais do que nunca tema de inúmeras publicações. É, desde 2003, prioridade da Organização Mundial de Saúde e, em 2004, foi reforçada pela Aliança Mundial para a Segurança do Paciente, que tem como objectivo principal facilitar o desenvolvimento de práticas e políticas de segurança do paciente a nível mundial. ${ }^{1,2}$

A criação da uma aliança mundial baseou-se na pressuposto de que, no futuro, uma má experiência com um doente em determinado local possa vir a ser fonte de aprendizagem e prevenção da mesma experiência em pacientes

* Médica de Família. USF Garcia de Orta - ACES Porto Ocidental de outros locais. O primeiro passo para tornar esta visão em realidade é assegurar que as intervenções e soluções de sucesso em determinada área se tornam conhecidas, compreendidas e aplicáveis no resto do mundo, uma vez que a base para a sua replicação se estabeleça.

O conceito de «segurança do paciente» implica que os profissionais envolvidos nos cuidados de saúde se asseguram permanentemente que os tratamentos e intervenções que os seus doentes recebem não lhes causam danos, lesões ou complicações além dos consequentes à evolução natural da sua própria doença e dos necessários e justificados para o diagnóstico e tratamento da mesma.

Despendemos mais tempo do nosso tempo de trabalho a responder a necessidades geradas pelas deficiências da organização dos cuidados, como a inexistência de processos de actuação ou a correcção de falhas na estrutura, do que a evitá-las, embora os nossos cuidados sejam idealmente centrados no utente e na sua família.

De forma simplista, digamos que uma organização centrada na segurança assegura-se que acontece ao doente $o$ que deveria acontecer e não acontece o que não deveria acontecer. 
Em Portugal, só muito recentemente começamos a preocupar-nos com a detecção e prevenção de eventos adversos nos serviços de saúde., ${ }^{3,4}$ À semelhança do que se tem passado noutros países, a maioria das intervenções conhecidas baseia-se em procedimentos hospitalares, dado que os riscos em meio hospitalar são mais elevados e potencialmente mais graves e as estratégias para a sua prevenção têm sido mais bem documentadas. Internacionalmente, os escassos estudos em cuidados primários de saúde centram-se sobretudo no erro e não nos eventos adversos.

Mas os eventos adversos em todas as suas vertentes ocorrem fora do ambiente hospitalar, nomeadamente em Cuidados de Saúde Primários. O momento de implementação de processos de notificação, estudo, prevenção e estabelecimento de uma cultura de segurança entre os nossos profissionais foi há muito ultrapassado, correndo nós, médicos de família e profissionais dos Cuidados de Saúde Primários, o risco de estarmos perigosamente desactualizados neste campo.

A proposta de um Sistema Nacional de Notificação de Incidentes e Eventos Adversos para a Segurança do Doente pela Direcção Geral da Saúde é ainda um projecto em concepção que urge concluir rapidamente.

Até lá, quer individualmente, quer em grupo, os médicos de família e as equipas em que se integram devem tomar nas suas mãos este problema.

\section{O DESENVOLVIMENTO DE UMA CULTURA DE SEGURANÇA}

Para entender e prevenir o erro, é necessário entender como acontece e, antes disso, dar conta que aconteceu. Isto requer mecanismos de identificação, compreensão e notificação de erro, bem como uma cultura que valoriza a vertente da aprendizagem em detrimento da culpabilização ou da punição.

Algumas características dos cuidados em Medicina Familiar predispõem à ocorrência de falhas e devem servir como campo de aplicação de procedimentos de segurança. ${ }^{5}$ Nestas incluem-se os ambientes de trabalho incertos e em constante mudança, as múltiplas fontes de informação por vezes contraditórias, as imprecisões, a necessidade de processar informação actualizada em circunstâncias e situações que mudam rapidamente, a presença da incerteza no diagnóstico, a importância de actuar por vezes de forma imediata, os momentos de intenso stress intercalados com longos períodos de activi- dade rotineira e repetitiva, a variabilidade individual e o ambiente de trabalho altamente influenciado por normas grupais e pela cultura organizacional ou a falta dela. ${ }^{6,7}$

Por esse motivo, as Unidades de Saúde devem, em primeiro lugar, estabelecer a rotina de identificação dos componentes necessários à criação da uma cultura de segurança do utente e que incluem:

a) a identificação das intervenções cuja implementação traz benefícios evidentes à prevenção de ocorrência de eventos adversos;

b) a discussão e acordo sobre as indicações para cada intervenção;

c) a aprendizagem e interiorização de procedimentos e técnicas para cada intervenção;

d) a avaliação periódica da aplicação dos procedimentos e suas consequências;

e) a introdução de novas práticas de acordo com os resultados obtidos.

Paralelamente às práticas diárias, um segundo componente, essencial para o desenvolvimento desta cultura, diz respeito à identificação, notificação, análise individual e em grupo e divulgação interpares de soluções para eventos adversos ocorridos na prática diária, nomeadamente os eventos com consequências graves para o doente.

No ano 2005, a Rede Médicos Sentinela procedeu à notificação voluntária de eventos adversos ocorridos nos centros de saúde. Este estudo teve como objectivos identificar o tipo e características de eventos adversos relacionados com os serviços de saúde, determinar a taxa estimada de incidência dos mesmos e desenvolver instrumentos de prevenção e redução de eventos adversos relacionados com os serviços de saúde.

Apesar de profissionais altamente motivados para a notificação, apenas $28 \%$ dos médicos participantes notificou, ao longo de um ano, um ou mais eventos adversos, num total de 208 notificações. A análise dos dados mostrou uma taxa superior de notificação de eventos relacionados com os médicos, sobretudo com danos psicológicos e materiais para os utentes. Este estudo revelou que, mesmo em médicos altamente motivados, os eventos adversos não estão enraizados como episódios de alerta para a melhoria da nossa prática clínica e redução dos riscos para os doentes. O desconhecimento da sua frequência e características deixa-nos fragilizados perante a sua prevenção. Esta situação não é, no entanto, característica isolada do nosso país. ${ }^{8,9}$ 
No entanto, vão sendo cada vez mais frequentes estudos sobre a ocorrência de eventos adversos em ambiente de Cuidados de Saúde Primários. Um caso próximo e recente é o estudo APEAS, realizado em Espanha e divulgado em 2008, que teve como objectivos melhorar o conhecimento sobre a magnitude, impacto e transcendência dos eventos adversos em Cuidados de Saúde Primários e aumentar o número de profissionais envolvidos na cultura de segurança do doente, entre outros. ${ }^{10}$ Este estudo revelou que, num universo de 96.047 doentes e 452 profissionais, se identificaram 18,63\%o (por mil) eventos adversos. Generalizando os resultados, os autores concluíram que, por ano, 7 em cada 100 cidadãos poderia ser afectado por um evento adverso relacionado com os cuidados de saúde.

Este resultado é significativo se tivermos em conta o número de contactos que os utentes estabelecem por ano com as Unidades de Saúde em Portugal. Só a identificação e estudo sistemático do tipo de eventos adversos mais frequentes e das formas de os evitar nos permitirá evoluir para a um processo racional de prevenção dos mesmos. Para tal, a existência de uma taxonomia universal do tipo de eventos é um instrumento de enorme utilidade.

\section{ÁREAS CHAVE DE INTERVENÇÃO EM CUIDADOS DE SAÚDE PRIMÁRIOS}

É importante adoptar medidas individuais e organizacionais de forma a evitar eventos adversos. Estas devem incluir, para além da prevenção, a ajuda e suporte emocional para o profissional que cometeu o erro. ${ }^{11}$

Também é essencial que cada médico reaja de forma adequada após o evento, analisando o processo que conduziu ao evento adverso para o doente, individualmente, em equipa e com a vítima (Quadro I).

A taxonomia dos eventos adversos envolve a identificação, classificação e descrição das razões pelas quais as falhas ocorrem num serviço de saúde e pode contribuir para elencar estratégias preventivas ou de minimização da incidência de eventos adversos no futuro de forma sistemática e organizada. As próprias definições de erro médico, evento adverso, incidente, acidente, quase-falhas devem ser esclarecidas para a melhoria da comunicação entre todos os que se interessam e preocupam com o tema. ${ }^{12,13}$

O que não se regista não existe. A notificação sistemática, voluntária, anónima e, no caso de eventos fatais, obrigatória, é o passo seguinte no caminho para a seguran-

QUADRO I. Áreas-chave de intervenção em Cuidados
de Saúde Primários
Taxonomia - classificação universal do tipo de eventos
adversos
Informação e treino de estudantes e profissionais em
Segurança do Doente
Desenvolvimento de sistema de notificação de eventos
adversos
Avaliação das causas e consequências
Identificação de factores-chave de mudança, generalizáveis
a todas as Unidades de Saúde
Implementação de procedimentos e normas de prevenção
futura
Incentivo e reconhecimento de Unidades que implementam
práticas de Segurança do Doente

ça do doente. A notificação permite a avaliação individual e em equipa do «caminho percorrido pelo erro», a identificação dos factores causais e das mudanças necessárias e a implementação das medidas correctoras que permitam a prevenção da repetição do mesmo evento adverso.

Dentro de cada área de intervenção é possível aplicar soluções para as causas mais frequentes de erro (Quadro II)..$^{14,15,16,17}$

\section{CONCLUSÃO}

Em Portugal, a segurança do doente e a notificação e resolução de eventos adversos mais do que uma necessidade, deve transformar-se numa cultura entranhada em cada profissional de saúde seja qual for o nível a que contacta com o utente dentro do sistema.

A segurança do doente é um tema abrangente, que implica empenhamento político e organizativo dos profissionais e dos doentes bem como de todos os intervenientes no processo de contacto dos utentes com o sistema de saúde. É urgente implementar mudanças que promovam esta cultura e tornem todas as actividades de cuidado aos doentes, desde a entrada até à saída, seguras e redutoras do risco de sofrer eventos adversos.

Ao nível dos Cuidados de Saúde Primários este processo é ainda mais urgente, dado que todas as intervenções se têm centrado nos cuidados de segunda linha, nomeadamente os cuidados cirúrgicos. No âmbito dos Agrupa- 


\begin{tabular}{|c|c|c|}
\hline & Causas & Soluções possíveis \\
\hline Medicamento & $\begin{array}{l}\text { Troca de medicação } \\
\text { Interacção medicamentosa grave } \\
\text { Duplicação de princípios activos iguais ou } \\
\text { sobreponíveis na sua acção } \\
\text { Atraso no início de tratamento } \\
\text { Troca de identificação de doente }\end{array}$ & $\begin{array}{l}\text { Normalizar apresentação da informação sobre o medicamento } \\
\text { Evitar medicamentos de aspecto e nome parecidos } \\
\text { Promover medidas de segurança na aplicação de injectáveis } \\
\text { Conciliar os tratamentos entre níveis de cuidados } \\
\text { Introduzir alertas de segurança nas aplicações informáticas } \\
\text { de prescrição } \\
\text { Desmaterializar a prescrição }\end{array}$ \\
\hline Diagnóstico & $\begin{array}{l}\text { Atraso ou ausência de diagnóstico } \\
\text { Não realização de procedimento de rastreio } \\
\text { Atraso ou ausência de avaliação de } \\
\text { resultados de exames } \\
\text { Interpretação incorrecta de resultados } \\
\text { Vieses de decisão } \\
\text { Troca de identificação de doente }\end{array}$ & $\begin{array}{l}\text { Introduzir alertas e follow-ups de resultados anormais } \\
\text { Normalizar a nomenclatura e interpretação dos exames } \\
\text { Disponibilizar sistemas de apoio à decisão }\end{array}$ \\
\hline Comunicação & $\begin{array}{l}\text { Troca de identificação de doente } \\
\text { Atraso ou falha na comunicação com o } \\
\text { doente } \\
\text { Comunicação ineficaz entre médico-paciente } \\
\text { quanto ao diagnóstico ou medicação }\end{array}$ & $\begin{array}{l}\text { Minimizar o uso de instruções verbais ou telefónicas } \\
\text { Treinar e recertificar periodicamente os profissionais em } \\
\text { entrevista clínica } \\
\text { Verificar sistematicamente a identidade dos doentes, cruzando } \\
\text { mais do que um dado de identificação (ex: nome e data de } \\
\text { nascimento), encorajando o doente a participar activamente no } \\
\text { processo } \\
\text { Partilhar experiências de quase-erro, eventos adversos e } \\
\text { eventos-sentinela } \\
\text { Fornecer informação clara e escrita sempre que possível } \\
\text { Implementar registo clínico único }\end{array}$ \\
\hline Organização & $\begin{array}{l}\text { Circuito do doente dentro do sistema } \\
\text { Falta de procedimentos normalizados para } \\
\text { todas as intervenções desde a entrada até à } \\
\text { saída do doente }\end{array}$ & $\begin{array}{l}\text { Estabelecer procedimentos de avaliação e prevenção de erro em } \\
\text { todo o circuito do doente dentro do sistema de saúde } \\
\text { Testar os procedimentos com simulação de casos } \\
\text { Actualizar os procedimentos periodicamente de forma a } \\
\text { incorporar as técnicas mais seguras e o conhecimento científico } \\
\text { mais actual }\end{array}$ \\
\hline Registo & $\begin{array}{l}\text { Ausência de registo } \\
\text { Troca de identificação de doente }\end{array}$ & $\begin{array}{l}\text { Introduzir sistemas de apoio à decisão, alertas ou passos } \\
\text { limitantes nos programas de registo clínico }\end{array}$ \\
\hline Capacitação & $\begin{array}{l}\text { Não valorização do risco de erro por parte } \\
\text { de profissionais e utentes } \\
\text { Desconhecimento de medidas de prevenção }\end{array}$ & $\begin{array}{l}\text { Desenvolver redes de comunicação entre profissionais e entre } \\
\text { doentes } \\
\text { Capacitar os doentes e o público em geral como parceiros no } \\
\text { processo de cuidados de saúde, incluindo os doentes nas } \\
\text { medidas que contribuem para a sua própria segurança }\end{array}$ \\
\hline Notificação & $\begin{array}{l}\text { Desconhecimento da existência de eventos } \\
\text { adversos em circunstâncias semelhantes e } \\
\text { respectivas soluções }\end{array}$ & $\begin{array}{l}\text { Propor instrumentos para detectar os eventos adversos } \\
\text { realçando a importância de uma cultura de não culpabilização e } \\
\text { de incentivo à notificação } \\
\text { Implementar um sistema nacional de notificação de eventos } \\
\text { adversos, voluntário e obrigatório para os eventos graves, não } \\
\text { culpabilizante } \\
\text { Implementar rotinas de partilha e discussão de eventos } \\
\text { adversos dentro das equipas } \\
\text { Implementar sistemas de registo de incidentes dentro das } \\
\text { equipas (ex. Diário de Bordo, SAAR*) } \\
\text { Premiar as organizações que incluem na sua prática a } \\
\text { identificação, notificação, avaliação e implementação de } \\
\text { medidas de correcção de eventos adversos }\end{array}$ \\
\hline
\end{tabular}

*SAAR - Situação, Antecedentes, Avaliação, Recomendação. 
mentos de Centros de Saúde, a implementação de um sistema monitorização e de prevenção de eventos adversos deveria ser considerado um indicador de boas práticas e, provavelmente, no futuro, um factor de contratualização com os seus clientes directos: os cidadãos. Os Cuidados de Saúde Primários portugueses, através das Unidades de Saúde que prestam directa ou indirectamente cuidados aos utentes, devem tornar-se, pois, organizações aprendentes, desculpabilizantes e não punitivas em tudo o que se relaciona com a ocorrência de eventos adversos.

\section{REFERÊNCIAS BIBLIOGRÁFICAS}

1. World Health Organization (WHO), Department of Health Service Provision. Towards a common international understanding of patient safety concepts and terms: taxonomy and terminology related to medical errors and system failure. Report of a WHO Working Group Meeting, Geneva, 8-11 October 2003. Disponível em: http://www.who.int/patientsafety/taxonomy/WHOTaxonomyConsultationOct2003.pdf [acedido em 26/10/2010].

2. World Health Organization (WHO).World Alliance for Patient Safety: forward programme. WHO Library Cataloguing-in-Publication Data, 2004. Disponível em: http://www.who.int/patientsafety/en/brochure_ final.pdf [acedido em 26/10/2010].

3. Ribas MJ. O erro em Medicina: contributo para a melhoria da qualidade. In: Valente Alves M, Ramos V. editores. Medicina Geral e Fami-liar: Da Memória. Lisboa: MVA Invent; 2003. p. 152-61.

4. Fragata J, Martins L. O Erro em medicina: perspectivas do indivíduo, da organização e da sociedade. Lisboa: Almedina; 2008.

5. Pedreira M, Harada M. A segurança do paciente. S. Caetano do Sul: Yendis Editora; 2009.

6. Reason J. Safety in the operating theatre - part 2: human error and organisational failure. Qual Safe Health Care 2005 Feb; 14 (1): 56-60.

7. Reason J. Combating omission errors through task analysis and good reminders. Qual Safe Health Care 2002 Mar; 11 (1): 40-4.

8. Borrell-Carrió F, Páez Regadera C, Suñol Sala R, Orrego Villagan C, Gil Terrón N, Martí Nogués M. Errores clínicos y eventos adversos: percepción de los médicos de atención primaria. Aten Primaria 2006 Jun; 38 (1): 25-32.

9. Moskowitz EJ, Nash DB.The quality and safety of ambulatory medical care: current and future prospects. Am J Med Qual 2007 Jul-Aug; 22 (4): 27488.

10. Estudio APEAS: estudio sobre la seguridad de los pacientes en Atención Primaria de Salud. Madrid: Ministerio de Sanidad y Consumo; 2008.

11. Fisseni G, Pentzek M, Abholz HH. Responding to serious medical error in general practice: consequences for the GPs involved: analysis of 75 cases from Germany. Fam Pract 2008 Feb; 25 (1): 9-13.

12. Elder NC, Pallerla H, Regan S. What do family physicians consider an error? A comparison of definitions and physician perception. BMC Fam Pract 2006 Dec 8; 7: 73

13. World Alliance for Safer Health Care. Conceptual Framework for the International Classification for Patien Safety. Final Technical Report. Geneva:World Health Organization; 2009.

14. Budnitz DS, Layde PM. Outpatient drug safety: new steps in an old direction. Pharmacoepidemiol Drug Saf 2007 Feb; 16 (2): 160-5.

15. Coulter A. After Bristol: putting patients at the centre. BMJ 2002 Mar 16; 324 (7338): 648-51.

16. Joint Comission Resources. Patient safety standards approved for home care, ambulatory care. Jt Comm Perspect 2003 Jul; 23 (7) :6-11.

17. WHO Collaborating Centre for Patient Safety Solutions. Patient Safety Solutions. Geneva:World Health Organization; 2007.

\section{CONFLITOS DE INTERESSE}

O autor declara não existirem conflitos de interesse na elaboração deste artigo

\section{ENDEREÇO PARA CORRESPONDÊNCIA}

Maria José Ribas

USF Garcia de Orta - ACeS Porto Ocidental

Rua Pinho Leal, 29, Foz do Douro, 4150-620 Porto

4100-038 Porto

E-mail: mariajribas@gmail.com

Recebido em 10/12/2010

Aceite para publicação em 30/12/2010

\section{ABSTRACT}

\section{ADVERSE EVENTS IN PRIMARY CARE: PROMOTING A CULTURE OF PATIENT SAFETY}

Patient safety has not been systematically included as a priority in the organization of care in Portuguese primary care health units. The concept of patient safety is not new and includes a group of activities designed to avoid adverse events in primary care. These adverse events include harm to patients, near misses, incidents, accidents and errors. Adverse events are caused by multiple factors and can be prevented. In order to do that, we need to know how anticipate and prevent these events. When they occur, we must be willing to report them, discuss them, share our experiences with others, and learn from them. Portuguese primary care requires a change towards a culture of safety which includes every act of care for the patient from the time of their entry until the time of their exit from the health care system.

Keywords: Adverse Events; Patient Safety; Medical Errors; Primary Health Care. 